\title{
Lack of Association between the COMT rs4680 Polymorphism and Ovarian Cancer Risk: Evidence from a Meta-analysis of 3,940 Individuals
}

\author{
Jin-Ze Du ${ }^{1}$, Yu-Ling Dong ${ }^{1}$, Guo-Xing Wan ${ }^{1,2}$, Lin Tao ${ }^{1}$, Li-Xia Lu${ }^{1}$, Feng Li ${ }^{1}$, \\ Li-Juan Pang ${ }^{1 *}$, Wei Jia ${ }^{1 *}$
}

\begin{abstract}
Catechol-O-methyltransferase (COMT) is involved in estrogen metabolism and is vital to estrogen-induced carcinogenesis, including that of ovarian cancer. Although many recent epidemiologic studies have investigated associations between the COMT rs 4680 polymorphism and ovarian cancer risk, the results remain inconclusive. We therefore performed a meta-analysis to derive a more precise estimate of associations. Systematic searches of the PubMed, Embase, Web of Science, Cochrane Library, Wanfang, China National Knowledge Infrastructure, and Chinese Biomedicine databases were undertaken to retrieve eligible studies. Odds ratios (ORs) with their corresponding $95 \%$ confidence intervals (CIs) were pooled to assess the strength of the association. In total, 8 case-control studies involving 1,293 cases and 2,647 controls were included in the meta-analysis. Overall, the results showed no evidence of significant association between the COMT rs4680 polymorphism and ovarian cancer risk in any of the assessed genetic models. Subgroup analyses by ethnicity also did not reveal any significant association in any genetic model $(p>0.05)$. In conclusion, our findings suggest that the COMT rs4680 polymorphism may not contribute to the risk of ovarian cancer.
\end{abstract}

Keywords: COMT, polymorphism, genetic, ovarian cancer, meta - analysis

Asian Pac J Cancer Prev, 15 (18), 7941-7945

\section{Introduction}

There has been considerable academic focus on the etiology of ovarian carcinoma, which has poor recurrencefree survival and overall survival and which is the third most common gynecological cancer worldwide (Schuler et al., 2014b; Schuler et al., 2014a). It is universally acknowledged that the primary diagnosed age, clinical stage, pathological type, histological grade and resistance to chemotherapy are critical prognostic factors for survival in cancer patients (Liu et al., 2014). There is substantial evidence supporting the hormonal influence on female tumors, such as breast and ovarian cancer, thereby underlining the importance of mechanisms underlying steroid biosynthesis in the pathogenesis of this disease (Jakubowska et al., 2010; Chan et al., 2014). It has been generally accepted that long-term, excessive exposure to circulating estrogen, which is metabolized to form catechol estrogen, contributes and may be causal to ovarian carcinogenesis. Estrogen is thought to exert its effects in ovarian cancer by enhancing the proliferation of ovarian epithelial cells and increasing their susceptibility to genetic errors during DNA replication (Goodman et al., 2001; Emori and Drapkin, 2014). Accordingly, variations in the genes involved in the biosynthesis and metabolism of estrogen may contribute to altered levels of estrogen and thereby contribute to the risk of ovarian cancer (Holt et al., 2007).

Catechol-O-methyltransferase (COMT) is an important estrogen-metabolizing enzyme involved in the conjugation and inactivation of the catechol metabolite of estrogen (Guldberg and Marsden, 1975). COMT is found at high levels in a variety of human tissues, including ovarian, breast, liver, and kidney tissues (Mannisto et al., 1992). Recent studies have found that a functional polymorphism, a single $\mathrm{G}$ to A base-pair change in COMT, results in the substitution of valine with methionine (COMT Val158Met, rs4680) at codon 108 in the gene encoding soluble COMT and at codon 158 of the gene encoding membrane-bound COMT (Mao et al., 2010). This change is believed to account for a three- to four-fold reduction in COMT activity (Weinshilboum and Raymond, 1977; Dawling et al., 2001). Several lines of evidence have suggested that the presence of this variant COMT could

${ }^{1}$ Department of Pathology, Shihezi University School of Medicine, Shihezi, ${ }^{2}$ Department of Oncology, The Third Affiliated Hospital of Hubei University of Medicine, Wuhan, Hubei, China*For correspondence: jiaweipatho@sina.com, ocean123456@163.com 
increase the risk of hormonally influenced cancers because of its decreased ability to inactivate catechol estrogens and by producing intermediate products of catechol estrogen metabolism (Hirata et al., 2008; Tian et al., 2014). Therefore, it is biologically reasonable to hypothesize that individuals with the low-activity COMT-A (COMT-Met) allele are more likely to be at higher risk of ovarian cancer owing to the formation and accumulation of carcinogenic catechol estrogens.

Several studies have attempted to address the association between the COMT rs4680 polymorphism and risk of ovarian cancer. However, the results of these studies remain inconclusive (Goodman et al., 2000; Goodman et al., 2001; Garner et al., 2002; Sellers et al., 2005; Holt et al., 2007; Delort et al., 2008). Moreover, other studies are usually unable to obtain a precise estimate of the genetic association for disease due to limited sample size and ethnic variability. Therefore, to overcome these limitations and reduce the likelihood of random errors resulting in false-positive or false-negative associations, we conducted a meta-analysis to assess accurately the association between the COMT rs4680 polymorphism and ovarian cancer risk. Our results suggested that this polymorphism might not be associated with ovarian cancer risk.

\section{Materials and Methods}

\section{Search strategy}

A comprehensive literature search of PubMed, Embase, Web of science, Cochrane Library, Wanfang, China National Knowledge Infrastructure, and Chinese Biomedicine databases was undertaken to retrieve relevant studies on the association of the COMT rs4680 polymorphism with ovarian cancer risk published through July 2014. Only human studies and articles in English and Chinese were included. The search strategy was based on various combinations of the following search terms: "COMT" or "Catechol-O-methyltransferase" or "Val158Met" or "rs4680", and "SNP" or "polymorphism" or "genotype" or "variant" and "ovarian cancer" or "ovarian carcinoma". Additional eligible studies were identified by manually searching bibliographies of original articles and review articles. In cases of overlapping data, only the most recent study or largest population was selected. Results from different ethnicities were viewed as separate studies in the meta-analysis.

\section{Selection criteria}

Studies were considered eligible if they met the following criteria: (1) the studies were case-control studies assessing the association between the COMT rs4680 polymorphism and ovarian cancer risk; (2) the studies provided sufficient information for estimating the odds ratios (ORs) with their 95\% confidence intervals (CIs); and (3) all diagnoses of ovarian cancer were pathologically or histologically confirmed. Publications were excluded if (1) the studies included no control population; (2) they constituted a duplication of previous studies; (3) they were abstracts, comments, letters, reviews, or editorial articles; or (4) the studies reported no outcomes or their reported outcomes were difficult to determine.

\section{Data extraction}

Information was extracted carefully from all eligible studies independently by two investigators according to the selection criteria listed earlier. Any disagreement was addressed by discussion or consultation with a third reviewer until a consensus was reached. For every eligible study, the following information was collected using a uniform tabulation: first author, year of publication, country, ethnicity, source of control, genotype method, sample size, number of genotypes, alleles in cases and controls, and evidence of HWE (Hardy-Weinberg equilibrium) in controls. Ethnicity was categorized as "Caucasian," "African," or "Mixed population." The "mixed population" category was used when an ethnic group's composition was unclear.

\section{Statistical analysis}

The strength of the association between the COMT rs4680 polymorphism and ovarian cancer risk was determined based of ORs with 95\% CIs using Review Manager version 5.2 software (provided by The Cochrane Collaboration, Oxford, UK; http://www.cochrane.org/ software/revman.htm). The pooled ORs were estimated for a dominant model (AA+GA vs GG), allele model (A vs G), homozygote model (AA vs GG), heterozygote model (GA vs GG), and recessive model (AA vs GA+GG) with an appropriate effect model chosen according to the heterogeneity between studies. Heterogeneity assumption was determined by the chi-square-based Q test (Higgins et al., 2003). A $p$-value of $<0.1$ indicated heterogeneity among studies, in which case the random effects model was used for the meta-analysis (Cochran, 1950; DerSimonian and Laird, 1986). Otherwise, the fixedeffects model was used (Mantel and Haenszel, 1959). I2 values were used to quantify heterogeneity (Higgins and Thompson, 2002). Stratified analyses were conducted by ethnicity, which was categorized into Caucasian and other populations (African or mixed populations). Sensitivity analyses were also carried out by sequential omission of individual studies under various comparisons. Moreover, potential publication bias was graphically estimated by Begg's funnel plot (Begg and Mazumdar, 1994), and the asymmetry was further assessed by Egger's linear regression test ( $p<0.05$ was considered significant) (Egger et al., 1997). The analyses for publication bias were performed using the Stata 12.0 software (Stata Corp., College Station, TX, USA).

\section{Results}

\section{Study characteristics}

Initially, 27 potentially relevant publications related to the association between the COMT rs4680 polymorphism and ovarian cancer risk were retrieved in the database search. After screening the titles and abstracts and performing a full-text review for potentially eligible articles, 6 articles were confirmed for inclusion in the meta-analysis in accordance with the selection criteria (Goodman et al., 2000; Goodman et al., 2001; Garner et al., 2002; Sellers et al., 2005; Holt et al., 2007; Delort et al., 2008). Since the publications of Holt et al. and Sellers 
et al. presented two case-control studies on different ethnicities, we viewed each ethnic population as a separate study in the pooled analysis (Sellers et al., 2005; Holt et al., 2007). Therefore, 8 case-control studies with a total of 1293 cases and 2647 controls were ultimately included in the meta-analysis. All the included studies were reported in English. Among them, 4 studies were on Caucasians, 2 on Africans, and 2 on mixed populations. The genotype distributions of the controls of all included studies were consistent with HWE except for the case-control study in an African population by Sellers et al. (Sellers et al., 2005). The detailed characteristics of the included studies are summarized in Table 1.
Meta-analysis results

The pooled ORs with their respective $95 \%$ CIs and the result of the heterogeneity test are presented in Table 2 and Figure 1. Overall, there was no evidence of a significant association between the COMT rs4680 polymorphism and ovarian cancer risk in any genetic model tested (AA+GA vs GG: $\mathrm{OR}=0.90,95 \% \mathrm{CI}=0.77-1.06$; $\mathrm{A}$ vs $\mathrm{G}$ : $\mathrm{OR}=0.95,95 \% \mathrm{CI}=0.85-1.05 ; \mathrm{AA}$ vs $\mathrm{GG}: \mathrm{OR}=0.90,95 \%$ $\mathrm{CI}=0.73-1.10$; GA vs GG: $\mathrm{OR}=0.92,95 \% \mathrm{CI}=0.77-1.10$; AA vs GA+GG: OR=0.95, 95\% CI=0.80-1.13). Similarly, no significant association was found in any genetic model (both $p>0.05$ ) in the subgroup analyses based on ethnicity as shown in Table 2.

Table 1. Main Characteristics of Studies Included in the Meta-analysis

\begin{tabular}{|c|c|c|c|c|c|c|c|c|}
\hline First author & Year & Country & Ethnicity & $\begin{array}{c}\text { Genotype-case } \\
\text { AA/GA/GG }\end{array}$ & $\begin{array}{c}\text { Genotype-control } \\
\text { AA/GA/GG }\end{array}$ & $\begin{array}{c}\text { Source } \\
\text { of control }\end{array}$ & $\begin{array}{l}\text { Genotype } \\
\text { method }\end{array}$ & HWE test \\
\hline Goodman (6) & 2001 & USA & Mixed & $16 / 57 / 52$ & $19 / 57 / 68$ & population & PCR-RFLP & $\mathrm{Y}$ \\
\hline Holt-b (7) & 2007 & USA & Caucasian & $76 / 129 / 72$ & $137 / 209 / 104$ & population & Taqman & $\mathrm{Y}$ \\
\hline Holt-a (7) & 2007 & USA & African & $10 / 19 / 04$ & $16 / 58 / 52$ & population & Taqman & $\mathrm{Y}$ \\
\hline Goodman (15) & 2000 & Germany & Caucasian & $27 / 54 / 27$ & $29 / 52 / 25$ & hospital & PCR-RFLP & $\mathrm{Y}$ \\
\hline Sellers-a (16) & 2005 & USA & Caucasian & $119 / 224 / 110$ & $147 / 269 / 127$ & hospital & PCR-RFLP & Y \\
\hline Sellers-b (16) & 2005 & USA & African & $17 * / 19$ & $30 * / 23$ & hospital & PCR-RFLP & NA \\
\hline Garner (17) & 2002 & USA & Mixed & $48 / 103 / 59$ & $54 / 119 / 52$ & population & PCR-RFLP & $\mathrm{Y}$ \\
\hline Delort (18) & 2008 & France & Caucasian & $18 / 22 / 11$ & $283 / 480 / 237$ & population & Taqman & $\mathrm{Y}$ \\
\hline
\end{tabular}

HWE, Hardy-Weinberg equilibrium; Y, in agreement with HWE test; NA, not available; *Number of patients with the AA+GA genotype in the case and control groups

Table 2. Meta-Analysis of the COMT rs4680 Polymorphism and Ovarian Cancer Risk

\begin{tabular}{lcccccc}
\hline $\begin{array}{l}\text { Genetic } \\
\text { contrast }\end{array}$ & $\begin{array}{c}\text { Sample size } \\
\text { (case/control) }\end{array}$ & OR $(95 \% \mathrm{CI})$ & $P$ & $\begin{array}{c}\text { Heterogeneity } \\
P_{h}\end{array}$ & $\mathrm{I}^{2}$ & $\begin{array}{c}\text { Effect } \\
\text { model }\end{array}$ \\
\hline AA+GA vs GG & $1293 / 2647$ & $0.90(0.77,1.06)$ & 0.22 & 0.62 & $0 \%$ & $\mathrm{~F}$ \\
Caucasian & $889 / 2099$ & $0.93(0.76,1.14)$ & 0.48 & 0.91 & $0 \%$ & $\mathrm{~F}$ \\
Other & $404 / 548$ & $0.85(0.64,1.13)$ & 0.26 & 0.21 & $34 \%$ & $\mathrm{~F}$ \\
A vs G & $2514 / 5188$ & $0.95(0.85,1.05)$ & 0.3 & 0.64 & $0 \%$ & $\mathrm{~F}$ \\
Caucasian & $1778 / 4198$ & $0.96(0.85,1.08)$ & 0.46 & 0.63 & $0 \%$ & $\mathrm{~F}$ \\
Other & $736 / 990$ & $0.92(0.75,1.13)$ & 0.43 & 0.3 & $17 \%$ & $\mathrm{~F}$ \\
AA vs GG & $658 / 1350$ & $0.90(0.73,1.10)$ & 0.31 & 0.9 & $0 \%$ & $\mathrm{~F}$ \\
Caucasian & $460 / 1089$ & $0.91(0.72,1.16)$ & 0.46 & 0.68 & $0 \%$ & $\mathrm{~F}$ \\
Other & $198 / 261$ & $0.85(0.56,1.29)$ & 0.45 & 0.72 & $0 \%$ & $\mathrm{~F}$ \\
GA vs GG & $949 / 1909$ & $0.92(0.77,1.10)$ & 0.34 & 0.55 & $0 \%$ & $\mathrm{~F}$ \\
Caucasian & $649 / 1503$ & $0.94(0.76,1.16)$ & 0.57 & 0.99 & $0 \%$ & $\mathrm{~F}$ \\
Other & $300 / 406$ & $0.84(0.50,1.40)$ & 0.5 & 0.1 & $57 \%$ & $\mathrm{R}$ \\
AA vs GA+GG & $1257 / 2594$ & $0.95(0.80,1.13)$ & 0.57 & 0.93 & $0 \%$ & $\mathrm{~F}$ \\
Caucasian & $889 / 2099$ & $0.95(0.79,1.15)$ & 0.62 & 0.59 & $0 \%$ & $\mathrm{~F}$ \\
Other & $368 / 495$ & $0.95(0.66,1.35)$ & 0.76 & 1 & $0 \%$ & $\mathrm{~F}$ \\
\hline
\end{tabular}

$P_{h}, p$-value for heterogeneity; I2, quantitative estimate for heterogeneity; $\mathrm{R}$, random effects model; $\mathrm{F}$, fixed effects model

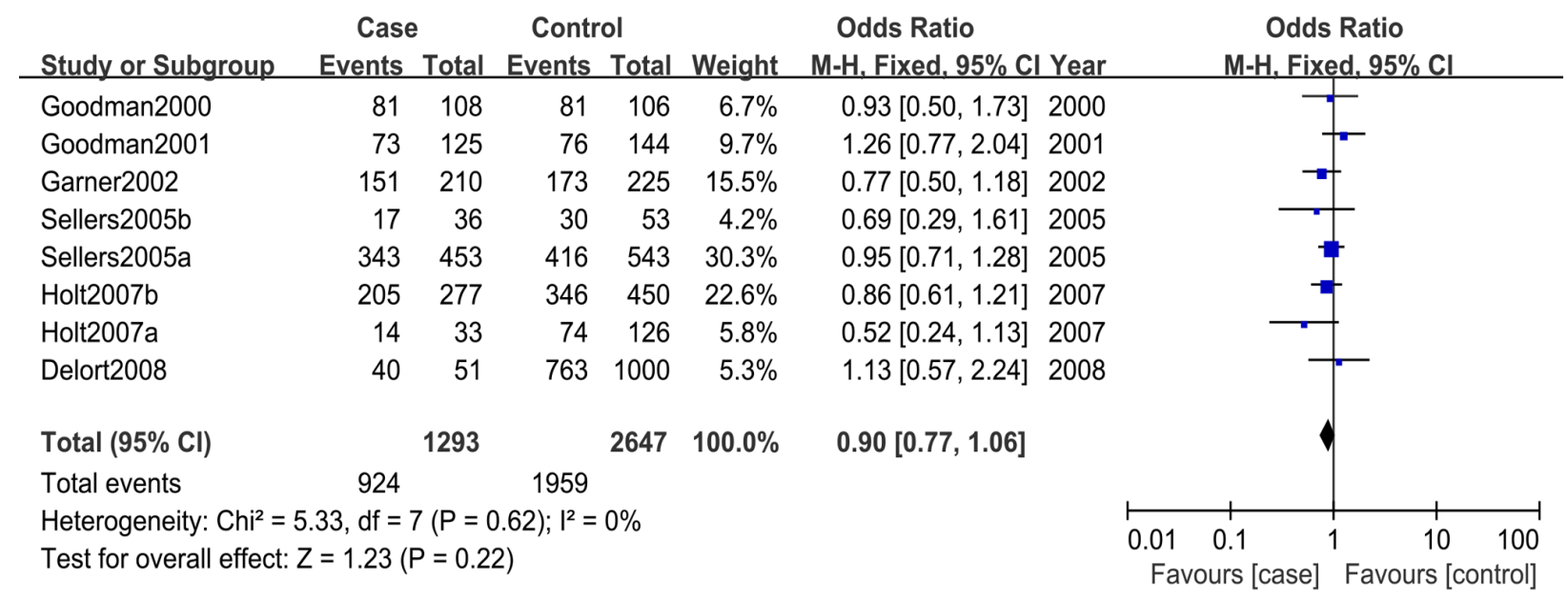

Figure 1. Forest Plot for the Association between the COMT rs4680 Polymorphism and Ovarian Cancer Risk Using a Fixed Effect Model (dominant model, AA+GA vs GG) 


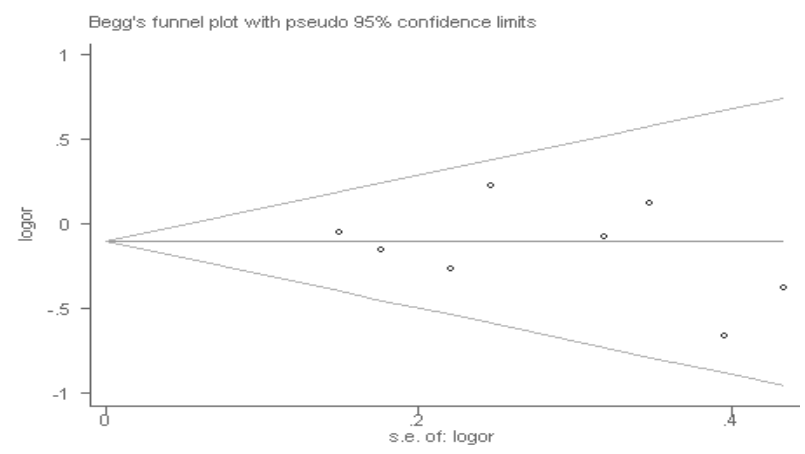

Figure 2. Begg's Funnel Plot for Publication Bias (dominant model, AA+GA vs GG)

\section{Publication bias and sensitivity analysis}

Begg's funnel plot analysis and Egger's test were performed to assess publication bias. The graphical funnel plot shown in Figure 2 is symmetrical (dominant model, AA+GA vs GG), indicating the absence of publication bias. In addition, no evidence of publication bias was revealed by the results of the Egger's test ( $p=0.51$ for the dominant model), indicating the robustness of our results. Sensitivity analyses were performed by sequential omission of individual studies to investigate the influence of each study on the pooled ORs. The corresponding pooled ORs were not consequently altered in any genetic model tested, reflecting the stability and reliability of the results.

\section{Discussion}

Our meta-analysis is the first to investigate the association between COMTrs4680 polymorphism and ovarian cancer risk, and our findings support the notion that there is no significant association between the two.

COMT, a phase II enzyme involved in estrogenmetabolism, is encoded by COMT located on chromosome 22q11 (Grossman et al., 1992). This enzyme plays a vital role in the development of hormonally influenced cancers, including ovarian cancer (Jakubowska et al., 2010). One possible underlying mechanism of COMT in cancer development is the inactivation of mutagenic metabolites of estrogens and transformation of catechol estrogens into non-genotoxic methyl ethers. Aberrant levels of COMT are thereby likely to accelerate the acquisition of a neoplastic phenotype (Guldberg and Marsden, 1975). As mentioned in the Introduction, a common single nucleotide polymorphism $(\mathrm{G} \rightarrow \mathrm{A})$ in codons 158 and 108 of the membrane-bound and cytosolic forms, respectively, results in a Val $\rightarrow$ Met amino acid substitution, which has been proven to be a determinant of COMT enzyme activity (Lachman et al., 1996; Dawling et al., 2001). It has been hypothesized that individuals carrying the low-activity COMT-A allele may be at increased risk of ovarian cancer owing to the resulting reduction in COMT enzyme activity (Goodman et al., 2000).

To date, several epidemiologic studies have been carried out to evaluate the association between the COMT rs4680 polymorphism and risk of ovarian cancer. However, the results remain inconclusive. For example, Delort et al. revealed a trend toward an increasing risk for developing ovarian cancer in women with the low-activity COMT genotype and allele, although the trend did not reach the statistical significance (Delort et al., 2008). Similar results were obtained by Goodman et al. (Goodman et al., 2001). Conversely, Garner et al. demonstrated that the rs4680 polymorphism was significantly associated with a decreased risk in mucinous tumors of the ovary, although the inverse association of the Val/Met variant was not evident in ovarian cancer overall (Garner et al., 2002). However, in the study by Holt et al., no association of ovarian cancer risk was observed with the COMT rs4680 polymorphism or in combination with variants of other relevant genes involved in estrogen-metabolism signaling (Holt et al., 2007).

In the current meta-analysis, there was no evidence showing a significant association between the COMT rs4680 polymorphism and ovarian cancer risk in any genetic comparison model tested. Moreover, the ORs in the pooled analyses under all comparisons trended toward a deceased risk of ovarian cancer with the COMT rs4680 polymorphism, although it was not significant. This finding was partially inconsistent with previous individual studies, suggesting the importance of sample size in genetic association studies. As an important estrogenmetabolism-related enzyme, the role of the COMT rs4680 polymorphism has been extensively studied in hormonally affected cancers. A meta-analysis by Wan et al. suggested that the COMT rs4680 variant might contribute to susceptibility to breast cancer in a Chinese population, particularly among premenopausal women (Wan et al., 2014). Lin et al. found no significant association of the COMT rs4680 polymorphism with endometrial cancer risk in the overall pooled analyses, but a significantly decreased risk in GA vs GG and AA+GA vs GG genetic models was noted in postmenopausal women (Lin et al., 2012). Furthermore, the study by He et al. showed that the COMT polymorphism was associated with a reduced risk of breast cancer in Caucasians but not in other populations (He et al., 2012). These findings may suggest important effects of menopausal status and ethnicity variation on the association of the COMT rs4680 polymorphism with hormonally affected cancers, which likely includes ovarian cancer. Our subgroup analyses by ethnicity did not reveal any significant associations in Caucasians or other populations. However, the true association between the COMT rs4680 polymorphism and ovarian cancer risk remains to be determined in Asians and Africans. Accordingly, future studies with larger sample sizes are warranted to verify this association in different ethnicities and considering menopausal status.

Some limitations need to be acknowledged when interpreting the results of our meta-analysis. First, regarding ethnicity, most of the included studies were conducted in Caucasians. Thus, it is of importance to ascertain the association using more studies and samples from other ethnicities for more accurate conclusions. Second, the role of the COMT rs4680 polymorphism in hormonally affected cancers has been frequently reported to be associated with menopausal status in women (Lin et al., 2012; Wan et al., 2014), which was not considered by most studies included in this analysis and thereby limits 
the subgroup analysis. Third, potential interactions of the COMT rs4680 variants with other important genetic polymorphisms involved in estrogen-metabolism that may influence the risk of ovarian cancer (Goodman et al., 2001), such as CYP1A1, CYP1A2, and CYP1B1 polymorphisms, in combination with environmental or hormonal exposures were not assessed because of the shortage of original data. Fourth, the unadjusted estimates were utilized in the meta-analysis because of data limitations.

In conclusion, the results of our meta-analysis suggest that the COMT rs4680 polymorphism may not be associated with the risk of ovarian cancer. However, studies including a larger sample size, diverse ethnic groups, well-matched cases and controls, and consideration of menopausal status are needed to further clarify the role of the COMT rs4680 polymorphism in ovarian cancer.

\section{Acknowledgements}

This work was supported by grants from the National Natural Science Foundation of China (No.81160316) and the Preeminent Youth Foundation of Shihezi University School of Medicine (No.2013ZRKXYQ-YD18). The funding agencies had no role in design, execution, and analysis of the study, or the decision to submit the paper for publication.

\section{References}

Begg CB, Mazumdar M (1994). Operating characteristics of a rank correlation test for publication bias. Biometrics, 1088-101.

Chan KK-L, Leung TH-Y, Chan DW, et al (2014). Targeting estrogen receptor subtypes (ER $\alpha$ and $\operatorname{ER} \beta$ ) with selective ER modulators in ovarian cancer. J Endocrinol, 221, 325-36.

Cochran WG (1950). The comparison of percentages in matched samples. Biometrika, 256-66.

Dawling S, Roodi N, Mernaugh RL, et al (2001). Catechol-Omethyltransferase (COMT)-mediated metabolism of catechol estrogens comparison of wild-type and variant COMT isoforms. Cancer Res, 61, 6716-22.

Delort L, Chalabi N, Satih S, et al (2008). Association between genetic polymorphisms and ovarian cancer risk. Anticancer Res, 28, 3079-81.

DerSimonian R, Laird N (1986). Meta-analysis in clinical trials. Control Clin Trials, 7, 177-88.

Egger M, Smith GD, Schneider M, et al (1997). Bias in metaanalysis detected by a simple, graphical test. $B M J, \mathbf{3 1 5}$, 629-34.

Emori MM, Drapkin R (2014). The hormonal composition of follicular fluid and its implications for ovarian cancer pathogenesis. Reprod Biol Endocrinol, 12, 60.

Garner EI, Stokes EE, Berkowitz RS, et al (2002). Polymorphisms of the estrogen-metabolizing genes CYP17 and catecholO-methyltransferase and risk of epithelial ovarian cancer. Cancer Res, 62, 3058-62.

Goodman JE, Lavigne JA, Hengstler JG, et al (2000). CatecholO-methyltransferase polymorphism is not associated with ovarian cancer risk. Cancer Epidemiol Biomarkers Prev, 9, 1373-6.

Goodman MT, McDuffie K, Kolonel LN, et al (2001). Casecontrol study of ovarian cancer and polymorphisms in genes involved in catecholestrogen formation and metabolism. Cancer Epidemiol Biomarkers Prev, 10, 209-16.

Grossman M, Emanuel B, Budarf M (1992). Chromosomal mapping of the human catechol- $<\mathrm{i}>\mathrm{O}</ \mathrm{i}>$-methyltransferase gene to 22q11. $\rightarrow$ q11. 2. Genomics, 12, 822-5.

Guldberg HC, Marsden CA (1975). Catechol-O-methyl transferase: pharmacological aspects and physiological role. Pharmacol Rev, 27, 135-206.

He X-F, Wei W, Li S-X, et al (2012). Association between the COMT Val158Met polymorphism and breast cancer risk: a meta-analysis of 30,199 cases and 38,922 controls. Mol Biol Rep, 39, 6811-23.

Higgins J, Thompson SG (2002). Quantifying heterogeneity in a meta-analysis. Stat Med, 21, 1539-58.

Higgins J, Thompson SG, Deeks JJ, et al (2003). Measuring inconsistency in meta-analyses. Bmj, 327, 557-60.

Hirata H, Hinoda Y, Okayama N, et al (2008). COMT polymorphisms affecting protein expression are risk factors for endometrial cancer. Mol carcinog, 47, 768-74.

Holt SK, Rossing MA, Malone KE, et al (2007). Ovarian cancer risk and polymorphisms involved in estrogen catabolism. Cancer Epidemiol Biomarkers Prev, 16, 481-9.

Jakubowska A, Gronwald J, Menkiszak J, et al (2010). BRCA1associated breast and ovarian cancer risks in Poland: no association with commonly studied polymorphisms. Breast Cancer Res Treat, 119, 201-11.

Lachman HM, Papolos DF, Saito T, et al (1996). Human catecholO-methyltransferase pharmacogenetics: description of a functional polymorphism and its potential application to neuropsychiatric disorders. Pharmacogenet Genomics, 6 , 243-50.

Lin G, Zhao J, Wu J, et al (2012). Contribution of catechol-Omethyltransferase Val158Met polymorphism to endometrial cancer risk in postmenopausal women: a meta-analysis. Genet Mol Res, 12, 6442-53.

Liu X-H, Man Y-N, Wu X-Z (2014). Recurrence season impacts the survival of epithelial ovarian cancer patients. Asian Pac J Cancer Prev, 15, 1627-32.

Mannisto P, Ulmanen I, Lundstrom K, et al (1992). Characteristics of catechol O-methyltransferase (COMT) and properties of selective COMT inhibitors. Prog Drug Res, 39, 291-350

Mantel N, Haenszel W (1959). Statistical aspects of the analysis of data from retrospective studies of disease. J Natl Cancer Inst, 22, 719-48.

Mao C, Wang X-W, Qiu L-X, et al (2010). Lack of association between catechol-O-methyltransferase Val108/158Met polymorphism and breast cancer risk: a meta-analysis of 25,627 cases and 34,222 controls. Breast Cancer Res Treat, 121, 719-25.

Schüler S, Lattrich C, Skrzypczak M, et al (2014a). Icb-1 gene polymorphism rs 1467465 is associated with susceptibility to ovarian cancer. J Ovarian Res, 7, 42.

Schüler S, Lattrich C, Skrzypczak M, et al (2014b). Polymorphisms in the promoter region of ESR2 gene and susceptibility to ovarian cancer. Gene, 546, 283-7.

Sellers TA, Schildkraut JM, Pankratz VS, et al (2005). Estrogen bioactivation, genetic polymorphisms, and ovarian cancer. Cancer Epidemiol Biomarkers Prev, 14, 2536-43.

Tian C, Liu L, Yang X, et al (2014). The Val 158 Met polymorphism in the COMT gene is associated with increased cancer risks in Chinese population. Tumor Biol, 35, 3003-8.

Wan G-X, Cao Y-W, Li W-Q, et al (2014). The Catechol-OMethyltransferase Val158Met Polymorphism Contributes to the Risk of Breast Cancer in the Chinese Population: An Updated Meta-Analysis. J Breast Cancer, 17, 149-56.

Weinshilboum RM, Raymond FA (1977). Inheritance of low erythrocyte catechol-o-methyltransferase activity in man. Am J Hum Genet, 29, 125. 